\title{
Mature and Immature Teratoma: A Report From the Second Italian Pediatric Study
}

\author{
Monica Terenziani, MD, ${ }^{1 *}$ Paolo $\mathrm{D}^{\prime}$ Angelo, $\mathrm{MD}^{2}{ }^{2}$ Alessandro Inserra, $\mathrm{MD},{ }^{3}$ Renata Boldrini, $\mathrm{MD},{ }^{3}$ \\ Gianni Bisogno, MD, ${ }^{4}$ Gian Luca Babbo, $\mathrm{MD}^{5}{ }^{5}$ Massimo Conte, $\mathrm{MD}^{6}{ }^{6}$ Patrizia Dall' Igna, $\mathrm{MD}^{4}{ }^{4}$ \\ Maria Debora De Pasquale, MD, ${ }^{3}$ Paolo Indolfi, $\mathrm{MD}^{7}{ }^{7}$ Luigi Piva, $\mathrm{MD}_{1}{ }^{1}$ Giovanna Riccipetitoni, $\mathrm{MD},{ }^{8}$ \\ Fortunato Siracusa, MD, ${ }^{9}$ Filippo Spreafico, MD, ${ }^{1}$ Paolo Tamaro, $\mathrm{MD}^{10}{ }^{10}$ and Giovanni Cecchetto, $\mathrm{MD}^{4}$
}

\begin{abstract}
Background. Teratomas demonstrate a benign clinical behavior, however they may recur with malignant components or as teratoma, and in a small group of patients prognosis could be fatal. After the first Italian study, we collected cases of teratoma, alongside the protocol for malignant germ cell tumors. Procedure. Patients with teratoma were collected from 2004 to 2014. Teratomas were classified according to the $\mathrm{WHO}$ classifications, as mature and immature. Patients with pathological aFP and/or bHCG, and those with a malignant germ cell component were not included. Results. The study enrolled 219 patients (150 mature, 69 immature teratomas) with a median age at diagnosis of 42 months. The primary sites involved were: 118 gonadal and 101 extragonadal teratomas. Two females with ovarian teratoma had a positive family history. Complete and incomplete surgeries were performed in $85 \%$ and
\end{abstract}

9\% of cases. Seventeen events occurred: six females had a second metachronous tumor (5 contralateral ovarian teratoma, 1 adrenal neuroblastoma) and 11 teratomas relapsed/progressed ( 3 mature, 8 immature teratomas). Two patients died, one of progressive immature teratoma and one of surgical complications. At a median follow up of 68 months, the event-free, relapse-free, and overall survival rates were $90.6 \%, 94.3 \%, 98.6 \%$, respectively. Conclusions. Teratomas show a good prognosis, especially the mature ones: surgery and follow-up remain the standard approach. Incomplete surgery in immature teratoma is the group at greatest risk of relapse. Bilateral ovarian tumors are a possibility, and the rare family predisposition to ovarian mature teratoma warrants further analyses. Pediatr Blood Cancer 2015;62:1202-1208. $\quad$ (C) 2015 Wiley Periodicals, Inc.

Key words: childhood; germ cell tumors; immature teratoma; mature teratoma

\section{INTRODUCTION}

Teratomas are the most common germ cell tumors in children and are classified as mature (MT), immature (IT), and malignant. IT contain neuroectodermal or blastemal tissue, the relative amount of which is scored according to a grading system introduced by Norris, modified by Gonzales-Crussi [1,2] and others $[3,4]$. Teratomas have a heterogeneous epidemiological, clinical, and pathological pattern in childhood and adolescence, relating to a different biological behavior of the disease. Teratomas develop from totipotent primordial cells, which have partly lost their genomic imprinting. MT is more frequent than IT; sacrococcygeal teratoma (SCT) occurs mainly in newborns and infants, with a female predominance; testicular teratoma (TT) in the first five years of life, and ovarian teratoma (OT) in older children up to 15 years of age [5,6]. Both the mature and the immature components are non-malignant tissues, and their metastatic potential is uncertain [2]. Despite the benign histological appearance of these lesions, there will be a small group of patients whose prognosis might be fatal [7].

Malignant cells can occur as other histological germ cell subtypes (semonoma, choriocarcinoma, embryonal carcinoma, and usually yolk sac tumor [YST] in children, or variable combination of these entities), or as a somatic differentiation called teratoma with a malignant somatic component (TMSC) [8]: in all such cases, it is the malignant tissue that decides the type of treatment. MT and IT usually demonstrate a benign clinical behavior, but they may recur with a malignant component or as a teratoma.

After the first Italian study [9], we developed guidelines for the treatment of teratoma, in conjunction with the second national prospective AIEOP (Associazione Italiana Ematologia Oncologia Pediatrica) study for malignant germ cell tumors, called the TCGM2004. A prospective collection of patients' data was activated, for the purpose of assessing prognostic factors, type of relapse and outcome.

\section{PATIENTS AND METHODS}

Patients with newly-diagnosed teratoma were collected from March 2004 to January 2014. The guidelines for teratoma had the approval of Ethical Committee. Central pathology review was available, but not mandatory and 153 out of $219(70 \%)$ were reviewed. Teratomas were classified according to the WHO classifications, as MT if they were characterized by differentiated tissue, IT if they contained immature, non-malignant tissues. Since IT could contain varying amounts of neuroectodermal or blastemal tissues, they were scored using a grading system introduced by Norris et al., modified by Gonzales-Crussi and others [1-4].

Initial diagnostic investigations, including medical history, physical examination, radiographic studies, histology, description of surgical specimen, serum levels of alpha fetoprotein $(\mathrm{aFP})$, and beta human chorionic gonadotropin (bHCG) were collected. In infants, serum aFP levels were monitored until they reached the normal value for the general population [10]. Patients with pathological serum levels of aFP and/or bHCG, and those with a

${ }^{1}$ Fondazione IRCCS Istituto Nazionale dei Tumori, Milano, Italy; ${ }^{2}$ ARNAS Civico, Ospedale Di Cristina e Benfratelli, Palermo, Italy; ${ }^{3}$ Ospedale Pediatrico IRCCS Bambino Gesù, Roma, Italy; ${ }^{4}$ University Hospital, Padova, Italy; ${ }^{5}$ Azienda Ospedaliera, Padova, Italy; ${ }^{6}$ Ospedale Giannina Gaslini, Genova, Italy; ${ }^{7}$ Seconda Università, Napoli, Italy; ${ }^{8}$ Ospedale dei Bambini V. Buzzi, Milano, Italy; ${ }^{9}$ Policlinico Universitario P. Giaccone, Palermo, Italy; ${ }^{10}$ Istituto IRCCS Burlo Garofalo, Trieste, Italy

Conflict of interest: Nothing to declare.

${ }^{*}$ Correspondence to: Monica Terenziani, Pediatric Oncology Unit, Fondazione IRCCS Istituto Nazionale dei Tumori, via Venezian 1, 20133 Milano, ITALY. E-mail: monica.terenziani@istitutotumori.mi.it Received 6 August 2014; Accepted 2 December 2014 
malignant germ cell component, including those with microscopic foci of YST, were not considered in the present analysis. Radiology, physical examination, and aFP were repeated every 3 and 4 months, respectively, in the first and second years after surgery, then every 6 months until the 5th year.

Primary surgical resection was the treatment indicated for all patients. The excision was considered complete (CS) if the teratoma had been resected in toto, with free margins according to the pathology report and no residual disease or rupture, or microscopically or macroscopically incomplete (IS), in the light of the pathology report and the surgeon's description. In cases of tumor rupture, the excision was defined as microscopically incomplete. Surgical biopsy was to be performed if excision of the mass was judged unfeasible. There were specific guidelines for OT, TT, SCT, and mediastinal teratoma. For OT, the surgical approach was via a transversal, subumbilical incision or a Pfannestiel incision as follows: excision of the tumor together with the ovary, preserving the salpinx, if possible; inspection of the contralateral ovary, biopsy of suspected areas or regional lymph nodes, peritoneal fluid sampling or washing for cytology. For TT, an inguinal orchifuniculectomy was recommended after clamping the spermatic cord. Complete en-bloc resection of the coccyx was mandatory for SCT. The guidelines contained no recommendations on laparoscopic procedures for ovarian masses and/or conservative surgery for TT: all these cases were collected nonetheless. The disease was not up-staged in the event of gliomatosis peritonei. Overall survival (OS), relapse-free survival (RFS) and event-free survival (EFS) rates were analyzed using the Kaplan-Meier method [11]. A logistic regression analysis was performed considering type of surgery (CS, IS), site of disease (OT, TT, SCT, other extragonadal T) and teratoma grade.

\section{RESULTS}

The study involved 219 patients, that is, 150 with MT and 69 with IT, with a median age at diagnosis of 42 months (range 0-20.4 years). The patients' main characteristics are listed in Table I. Centralized pathology review was done for $70 \%$ of patients, and the diagnosis was consistent in $95 \%$ of cases: the discrepancies mainly concerned MT with microscopic foci of immaturity being changed to IT grade I, IT grade II changing to grade III, and vice versa, and teratomas with microscopic foci of YST. There was a female prevalence of teratoma $(\mathrm{F}: \mathrm{M}=2.5: 1)$ and the primary sites involved were: 95 OT, $23 \mathrm{TT}$, and 101 extragonadal teratomas (ET). Eleven patients (5\%) had associated congenital malformations or syndromes (Table II).

\section{Mature Teratoma}

MT accounted for $68.5 \%$ of the whole series, with a median age of 55 months. We observed 68 OT, 63 ET, and 19 TT. Two patients developed other malignancies, a synchronous medulloblastoma and a metachronous adrenal neuroblastoma; both tumors were removed completely and the two patients were alive and relapse-free at the time of writing. Two patients with OT had a family history of OT in the mother.

CS were obtained at diagnosis in 133 patients (89\%), and IS in 8 $(5 \%)$. This information was unavailable for nine cases. One patient with SCT obtained a CS after two successive surgical procedures. One of two females with synchronous bilateral OT underwent bilateral enucleation, the other had oophorectomy and enucleation. Of 66 females with unilateral OT, seven had tumor enucleation (5 using a laparoscopic procedure), and two had laparoscopy and oophorectomy. The remainder $(87 \%)$ had oophorectomy according

TABLE I. Patient Characteristics

\begin{tabular}{|c|c|c|c|}
\hline & MT (150) & IT (69) & Total \\
\hline Sex F/M & $109 / 41$ & $48 / 21$ & $157 / 62$ \\
\hline \multicolumn{4}{|l|}{ Age } \\
\hline Range (years) & 0-20.4 & $0-15.2$ & $0-20.4$ \\
\hline Median (months) & 55 & 7 & 42 \\
\hline \multicolumn{4}{|l|}{ Primary site of $\mathrm{T}$} \\
\hline Ovary & 68 & 27 & 95 \\
\hline Testis & 19 & 4 & 23 \\
\hline Extra & 63 & 38 & 101 \\
\hline Sacrococcyx & 40 & 25 & 65 \\
\hline Mediastinum & 8 & 4 & 12 \\
\hline Retroperitoneum & 6 & 4 & 10 \\
\hline Other site & 9 & 5 & 14 \\
\hline \multicolumn{4}{|l|}{$\mathrm{T}$ grading } \\
\hline Not done & & 4 testes $+4 \mathrm{ET}$ & \\
\hline 1 & & 25 & 25 \\
\hline 2 & & 25 & 25 \\
\hline 3 & & 11 & 11 \\
\hline \multicolumn{4}{|l|}{ Surgery } \\
\hline Complete & 133 & 54 & 187 \\
\hline Incomplete & 8 & 11 & 19 \\
\hline Unknown & 9 & 4 & 13 \\
\hline Relapse & 3 & 8 & 11 \\
\hline Exitus & 0 & 2 & 2 \\
\hline Lost to follow-up & 4 & 3 & 7 \\
\hline Metachronous OT & 4 & 1 & 5 \\
\hline
\end{tabular}


TABLE II. Associated Syndrome

\begin{tabular}{|c|c|c|c|c|}
\hline & Site of teratoma & & Gender & Age at diagnosis \\
\hline \multicolumn{5}{|l|}{ Mature teratomas } \\
\hline 1 Down syndrome & retroperitoneal & & M & $4 \mathrm{mos}$ \\
\hline 1 Down syndrome & adrenal cavity & & $\mathrm{F}$ & connatal \\
\hline 1 partial deletion of chromosome 1 & $\mathrm{SC}$ & & $\mathrm{F}$ & connatal \\
\hline 1 three rays left hand agenesia & SC & & $\mathrm{F}$ & connatal \\
\hline 1 coccyx agenesia & $\mathrm{SC}$ & & $\mathrm{F}$ & connatal \\
\hline 1 monorchid & testis & & M & $16 \operatorname{mos}$ \\
\hline 1 interventricular shunt & ovary & & $\mathrm{F}$ & connatal \\
\hline 1 synchronous medulloblastoma & ovary & & $\mathrm{F}$ & 21 yrs \\
\hline 2 cases of mother with the same disease & ovary & & $\mathrm{F}$ & $14 / 11$ yrs \\
\hline \multirow[t]{2}{*}{1 metachronous neuroblastoma } & $\mathrm{SC}$ & & $\mathrm{F}$ & connatal \\
\hline & Site of Teratoma & Grade & Gender & Age at diagnosis \\
\hline \multicolumn{5}{|l|}{ Immature teratomas } \\
\hline 1 Currarino syndrome ${ }^{a}$ & $\mathrm{SC}$ & 2 & M & $22 \operatorname{mos}$ \\
\hline 1 lower limb congenital ectromelia & ovary & 2 & $\mathrm{~F}$ & 12 yrs \\
\hline 1 congenital hypothyroidism & $\mathrm{SC}$ & 3 & M & connatal \\
\hline 1 tricuspid valve atresia & SC & 2 & $\mathrm{~F}$ & $17 \mathrm{mos}$ \\
\hline 1 TMSC (diffuse carcinoma) ${ }^{\mathrm{a}}$ & SC & 2 & M (pt with Currarino S.) & $22 \mathrm{mos}$ \\
\hline 1 TMSC (1 microfocal localization of carcinoma) & $\mathrm{SC}$ & 2 & $\mathrm{~F}$ & connatal \\
\hline 1 TMSC (1 microfocal localization of medulloepithelioma) & ovary & 3 & $\mathrm{~F}$ & 14 yrs \\
\hline
\end{tabular}

SC, sacrococcyx; TMSC, teratoma with malignant somatic component. ${ }^{\text {a }}$ same patient.

to the surgical guidelines. Orchifuniculectomy was performed in 12 males, and 7 (37\%) had tumor enucleation with preservation of the residual testicular tissue. One male in this last group was monorchid at birth. No cases of bilateral TT were recorded.

Second tumors and relapses. Eight events were observed (4.6\%): four of the 66 patients with OT had contralateral metachronous OT, detected after 11-84 months of follow-up; they were managed by means of sparing surgery in three and ovariectomy in one. One female had a metachronous adrenal neuroblastoma. All these patients were alive and relapse-free at the time of writing. Three ET relapsed with YST and/or an increase in aFP, two of whom had undergone CS at diagnosis. One SCT with a recurrence in the vertebral canal was treated with two different platinum-based chemotherapy regimens to maximize response, without further surgery. A patient with head and neck teratoma had a biopsy at the time of relapse, followed by chemotherapy, with a normalization of the pathological aFP levels, and there was no evidence of vital tumor at subsequent surgery on the residual mass. The last patient was a female with an increased aFP and local and lung metastases. After biopsy she underwent cisplatin-based chemotherapy, and her aFP levels returned to normal, with complete remission of metastases, and partial remission of the presacral lesion, without further surgery. All three patients were alive, while two had stable disease after relapsing 10 and 115 months. The main clinical characteristics of the patients who relapsed are listed in Table III.

\section{Immature Teratoma}

Sixty-nine patients $(31.5 \%)$ had IT at a median age of 7 months. There was a prevalence of extragonadal sites (55\%), with 38 ET, 27 OT, and 4 TT. For $8 / 69$ cases ( $11.6 \%$ ), information on tumor grading was unavailable (because four were cases of TT [12], and the local pathologist had not graded the lesions in another four); among the other 61 IT, 25 were grade 1 (G1), 25 were grade 2 (G2), and 11 were grade 3 (G3).

Three patients had TMSC at diagnosis (Table II), one at relapse. The first three cases included one with a microfocal area of carcinoma, one with a microfocal area of medulloepethelioma (for which CS was the only treatment administered), and one male with Currarino syndrome and SCT G2 with multiple foci of carcinoma. After IS this male was administered a cisplatin-based chemotherapy. All patients were alive and relapse-free with a median time of follow-up of 36 months.

CS and IS were obtained at diagnosis in 54 patients (78\%) and 11 patients $(16 \%)$, respectively, while no information was available on four cases. Two patients with SCT achieved a CS after two successive surgical procedures, and one patient underwent biopsy for a mediastinal mass followed by a CS.

There were three OT with gliomatosis peritonei ( $1 \mathrm{G} 3,1 \mathrm{G} 2$, and $1 \mathrm{G} 1$ ), and three patients with OT G1 had tumor spillage: one of these during a laparoscopic enucleation. None of the OT with gliomatosis or tumor spillage relapsed. Of the 27 females with OT, 2 were treated with a laparoscopic procedure (1 oophrectomy and 1 tumor enucleation). The remainder $(92.6 \%)$ had oophorectomy according to the surgical guidelines.

Orchifunilectomy was performed in all four patients with TT; there were no cases of bilateral TT. Chemotherapy was administered at the discretion of treating physician in 4 cases.

Second tumors and relapses. Nine patients (13\%) experienced an event: eight teratomas relapsed, and one patient had a contralateral metachronous ovarian MT. In this last case, the tumor was detected after 36 months of follow-up; it was managed by means of sparing surgery and the patient was alive and relapse-free.

Any relapse or progression was always local: four teratomas relapsed with malignant YST and/or an increase in aFP, and four with a teratomatous component alone. $\mathrm{CS}$ at the time of diagnosis 


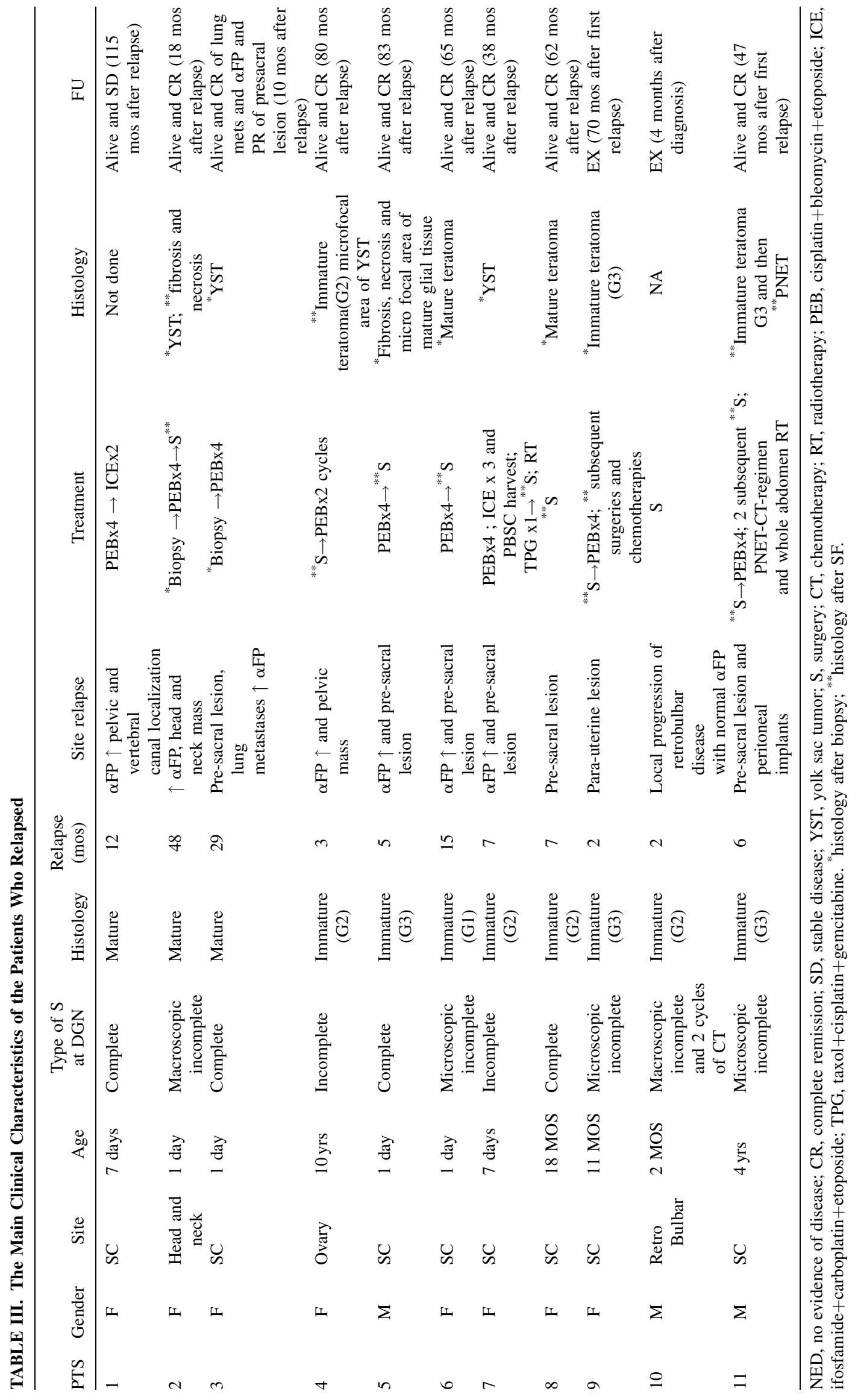


were 2 in the 54 patients whose primary resection had been complete, and incomplete in 6 of the 11 patients whose primary surgery had been IS. All but one of these eight patients had ET, that was graded as follows: $1 \mathrm{G} 1(4 \%) ; 4 \mathrm{G} 2(16 \%)$, and $3 \mathrm{G} 3(27 \%)$.

Among the malignant relapses, one female with OT revealed a pathological increase in aFP levels associated with a pelvic mass. She underwent complete excision of the recurrent tumor and aFP levels returned to normal. She was administered 2 cycles of cisplatin-based chemotherapy, with a follow up of 80 months after the relapse. Two other patients with SCT who experienced a pathological increase in their aFP levels and a presacral lesion were given platinum-based chemotherapy and surgery on the residual disease. One female with pathologically high aFP levels had multiple local relapses near the rectum: she was administered three different chemotherapy regimens and one attempt at surgery [13], which resulted in an IS and rapid tumor re-growth. A last attempt was made with radiotherapy, obtaining complete remission of the disease, with a follow up of 38 months after the relapse.

In the group that had exclusively teratomatous relapses, a SCT G2 relapsed after 7 months. The disease was treated with surgery and MT was found. There were 2 SCT G3 teratoma with multiple relapses. The first patient was a female with diagnosis at the age of 11 months who had microscopic residual disease after surgery. The teratoma relapsed showing the same histology that was partially removed. She underwent cisplatin-based chemotherapy, but 23 months later the tumor relapsed locally. After multiple relapses, and distant spread to the lungs, she died of progression 70 months from diagnosis. The second patient was a male whose teratoma was diagnosed at the age of 4 years. The tumor was removed leaving microscopic residual disease, that relapsed locally with peritoneal implants. After IS and platinum-based chemotherapy, the teratoma relapsed twice more, with two further attempts at surgery. Given his multiple peritoneal implants and the increasing neuroectodermal component, this was considered a TMSC with PNET transformation. The male was therefore treated according to the PNET protocol with chemotherapy and radiotherapy to the whole abdomen. He was alive and relapse-free at the time of writing. The last patient in this group was a male who underwent partial resection of a retrobulbar IT G2 followed by platinum-based chemotherapy, which was stopped after 2 cycles due to tumor progression with a decline in aFP to normal levels for the patient's age. Another attempt at surgery was made but the infant died of surgical complications. Two out of 69 patients (2.9\%) died, one of progressive disease and one of surgical complications. The main clinical characteristics of the patients who relapsed are listed in Table III.

All six patients with metachronous tumors were alive and relapsefree. Of the 11 patients who experienced a disease relapse, 7 were alive and in complete remission, 2 patients with IT died (one of progressive disease and the other of surgical complications), and 2 patients with MT were alive with stable disease but normal aFP levels, 115 and 10 months after relapsing. With a median follow-up of 68 months (range 0-153 months), the EFS, RFS, and OS rates were: $90.6 \%$, $94.3 \%, 98.6 \%$. Figures $1-3$ show the survival rates for whole series, MTand IT. The only factor of statistical significance emerging from the logistic regression was IS in the series as a whole $(P=0.015$; CI 1.45-25.94) and in IT group ( $P=0.04$; CI 1.18-39.42).

\section{DISCUSSION}

The novel insights generated by our data concern: the need to set up guidelines for the appropriate use of laparoscopy and

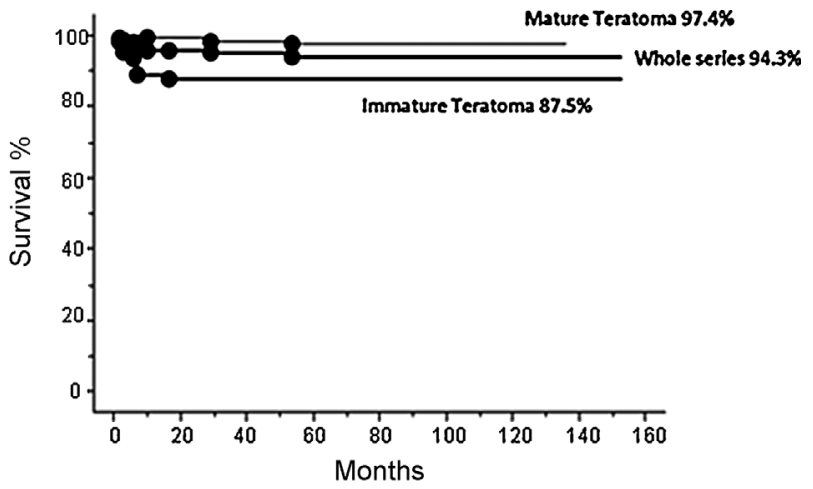

Fig. 1. Relapse-free survival after mature or immature teratoma, and in the whole series.

organ-sparing surgery in OT; the need for a prolonged follow-up due to the risk of contralateral OT, and the consequent use of available fertility-preserving techniques; the emerging evidence of familial forms that should be collected and studied.

The series of teratomas described here was registered after patients had been referred to pediatric surgery centers. It reveals an increase in the number of cases by comparison with our first study. Some of the findings reported here are consistent with other reports and already well known [5,7,14-17], i.e., the female and SC predominance, the ovarian localization seen in adolescents, the bimodal age distribution, and the association with congenital malformations and/or chromosomal syndromes. Our results confirm previous reports of a good overall survival rate for teratoma $[5,7,9,14-16]$. Efforts have been made to use chemotherapy as an adjuvant strategy for high-risk teratomas $[9,15,18]$, but the fundamental role of surgery as the gold standard is clear, and it is often the only treatment approach possible $[5,14,16,19,20]$. Patients in our series with microfocal TMSC and CS were given no additional therapies and, despite the limited number of patients involved, this result would give the impression that surgery alone might suffice in this subset of patients too.

There were no relapses or deaths among patients with TT. To preserve gonadal function in patients whose aFP and bHCG levels are within normal range, we recommend considering the conservative approach, where technically feasible.

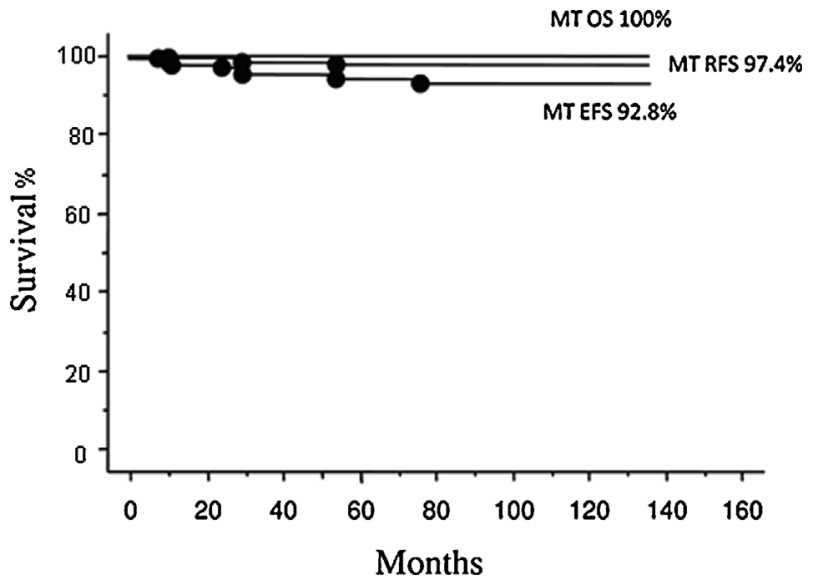

Fig. 2. Event-free survival, relapse-free survival, and overall survival after mature teratoma. 


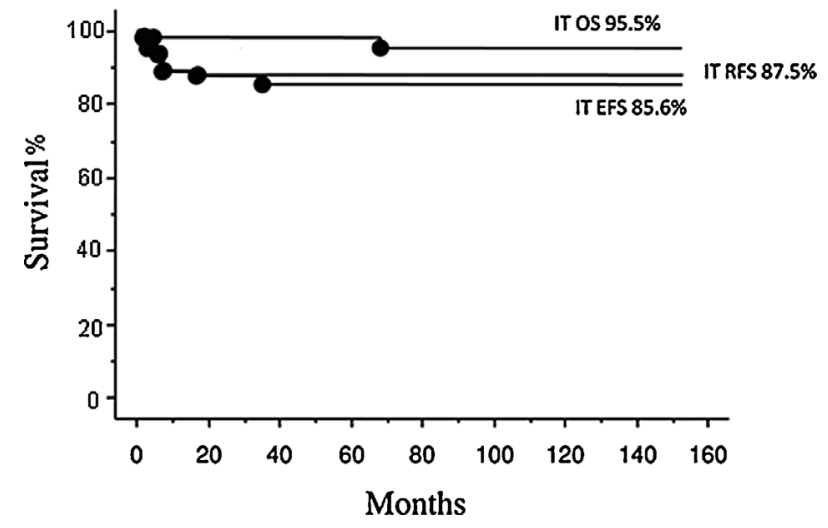

Fig. 3. Event-free survival, relapse-free survival, and overall survival after immature teratoma.

There were no deaths among OT patients. None of the OT with gliomatosis in our series relapsed: this finding is of interest because there have been reports of this condition being associated with a higher risk of relapse [5]. Larger series will need to be assessed to shed light on this issue. The use of laparoscopy is increasing, but its proper use in childhood ovarian tumors needs to be standardized (i.e., tumor dimension, cystic component) $[5,19,21]$. It is important to avoid the risk of tumor spillage and rupture, and the consequent up-staging of the disease. Conservative surgery to preserve gonadal function might be considered, where technically feasible, but only in patients whose aFP and bHCG levels are within normal range. Bilateral metachronous OT was seen in five patients, representing $5.2 \%$ of the whole series of OT. Although this percentage is low, this possibility is worth bearing in mind when scheduling patient follow-up. The risk of bilaterality is already known and ranges from 10 to $23 \%$ in women of all ages [22,23]. In our sample, the timing of the diagnosis of contralateral tumors ranged between 11 and 84 months, but the interval could be even longer. A three-year follow-up has been suggested for teratoma at any site [7,19,22]. It may be wise to extend the follow-up for females with OT given the risk of contralateral tumor, or to recommend regular yearly pelvic ultrasound throughout their fertile lives. The estimated growth rate of MT is slow, and the goal of repeated ultrasound is to preserve fertility [22]. Conservative ovarian surgery should be the standard indication for females for whom fertility is an important issue. To make this option possible, it is mandatory to ensure the viability of the remaining ovarian tissue without running the risk of incomplete tumor removal. Another possible option in these rare circumstances, depending on the patient's age, is to consider fertility preservation counseling.

Although MT are some of the most common ovarian neoplasms in females younger than 20 years of age, familial cases are very rare. Nezhat et al. [24] described a familial cystic ovarian teratoma and reviewed other familial cases (sisters, twins, mother, and daughters). The hypothesized pathogenesis implies a genetic defects that could be transferred from one generation to the next, giving rise to an increased risk in the same family [25]. All these findings point to the importance of collecting these data and gathering an exhaustive family medical history.

Survival rates show that the group with IT plus IS was more likely to recur. It has been reported that the risk of recurrence is higher for cases of IS, immature elements, and for SCT, than for gonadal sites. $[5,7,9,15,20,26]$. In the present series, the only statistically significant factor for relapse was IS in the series as a whole and in the IT group. The limitation of this study is that some of the findings reported here are consistent with other reports and already well known. The importance of CS is well-documented, and in some cases it is hard to achieve this without mutilating surgery. Nevertheless, it is important to ensure that all pediatric surgeons are well aware of the relevance of CS and, whenever possible, to suggest for a subsequent radical surgery. The present and previous Italian studies [9] identified much the same percentages of cases of IS ( $9 \%$ vs. $10 \%$ ), and of patients among them who relapsed (37\% vs. $35 \%$ ), confirming the high risk of relapse in this subset of patients. For all these reasons, it is important to perform follow up exams in patients with teratomas, but especially those incompletely resected with IT. In cases with a malignant germ cell component, aFP is an early sign of relapse, and testing aFP levels may help to reduce the burden of follow-up.

The standard treatment for relapse (surgery for teratoma and platinum-based chemotherapy for malignant germ cell components) was effective, but with some exceptions. For the rare condition of TMSC, the pathologist's expertise is fundamentally important in helping clinicians to choose the optimal treatment. There are no typical clinical or radiological signs to predict TMSC, which is one of the reasons why surgery or biopsy are advocated for patients without evidence of elevated tumor markers. In cases of multiple relapses with an aggressive behavior, transformation is a possibility to consider.

Another critical issue is the management of the disease with no evidence of malignant component [18]. In 1982 Gonzalez-Crussi reported on the risk of IT being classified as benign, but subsequently becoming metastatic [2]. Progressive tumor growth can prove fatal if surgery is not feasible because teratomas are scarcely sensitive to chemotherapy and radiotherapy. For a case of growing teratoma (GT) syndrome [28], in which surgery is not an option, it has been suggested that an attempt be made with four agent-drug combinations with an antiangiogenetic potential, as well as low-dose chemotherapy [29]. Whatever the underlying mechanism, and despite their biological diversity, teratomas, and GT syndromes share much the same therapeutic problems, since both are unresponsive to chemotherapy. On these grounds, an attempt at individual treatment with an antiangiogenetic potential might be worthwhile. For the time being, no effective drugs are currently available and an international effort would be helpful.

In conclusion, patients with teratoma have good survival rates and there appears to be no need for further treatment after surgery. IT with IS are at higher risk of relapse than those with MT (with or without a malignant component). New approaches are needed to deal with this last condition that, though rare, may sometimes be fatal. Several novel findings emerge from this study concerning: the feasibility of omitting chemotherapy even for patients with TMSC with CS; the lack of appropriate guidelines for the use of laparoscopy in pediatric patients; the need for a protracted follow-up due to the risk of contralateral OT (with a view to avoiding fertility-related consequences in young females); and a rare family predisposition for mature OT that warrants further analysis.

\section{REFERENCES}

1. Norris HJ, Zirken HI, Benson WL. Immature teratoma of the ovary: A clinical and pathological study of 58 cases. Cancer 1976;37:2359-2372. 
1208

Terenziani et al.

2. Gonzalez-Crussi. Extragonadal teratoma. Atlas of Tumor Pathology. 2nd series, Fascicle 18. Washington Armed Forces Inst of Pathology 1982. pp 44-129.

3. Dehner LP. 1986. Gonadal and extragonadal germ cell neoplasms. Teratoma in childhood. In: Finegold M Benington JL editor., Pathology of Neoplasia in Children and Adults. Philadelphia, PA, WB: Saunders. pp 282-312.

4. Heifetz SA, Cushing B, Giller R, Shuster JJ, Stolar CJ, Vinocur CD, Hawkins EP. Immature teratomas in children: Pathologic considerations: A report from the combined Pediatric Oncology Group/Children's Cancer Group. Am J Surg Pathol 1998;22:1115-1124.

5. Mann JR, Gray ES, Thornton C, Raafat F, Robinson K, Collins GS, Gornall P, Huddart SN, Hale JP, Oakhill A. Mature and immature extracranial teratomas in children: the UK Children's Cancer Study Group experience. J Clin Oncol 2008;21:3590-3597.

6. Harms D, Zahan S, Gobel U, Schneider DT. Pathology and molecular biology of teratomas in childhood and adolescence. Klin Padiatr 2006;218:296-302.

7. Derikx JP, De Backer A, van de Schoot L, Aronson DC, de Langen ZJ, van den Hoonaard TL, Bax NM, van der Staak F, van Heurn LW. Factors associated with recurrence and metastasis in sacrococcygeal teratoma. Br J Surg 2006;93:1543-1548.

8. Terenziani M, D'Angelo P, Bisogno G, Boldrini R, Cecchetto G, Collini P, Conte M, De Laurentis T, Ilari I, Indolfi P, Inserra A, Piva L, Siracusa F, Spreafico F, Tamaro P, Lo Curto. Teratoma with a malignan somatic component in pediatric patients: The Associazione Italiana Ematologia Oncologia Pediatrica (AIEOP) experience. Pediatr Blood Cancer 2010;54:532-537.

9. Lo Curto, D'Angelo M, Checchetto P, Klersy G, Dall' Igna C, Federico P, Siracusa A, Alaggio F, Bernini R, Conte G, De Laurentis M, Di Cataldo T, Inserra A, Santoro A, Tamaro N, Indolfi P. Mature and immature teratoma: results of the first paediatric Italian study. Pediatr Surg Int 2007;23:315-322.

10. Tsuchida Y, Endo Y, Saito S, Kaneko M, Shiraki K, Ohmi K. Evaluation of alpha-fetoprotein in early infancy. J Pediatr Surg 1978;13:155-156.

11. Kaplan EL, Meier P. Non parametric estimation from incomplete observations. J Am Stat Assoc 1958:53:457-481.

12. Ulbright TM, Amin MB, Young RH, editor., Atlas of Tumor Pathology, Tumors of the testis, adnexa, spermatic cord, and scrotum. AFIP Third series. Washington DC: Armed Forces Institute of Pathology. $1999258 \mathrm{p}$

13. Eggener SE, Carver BS, Loeb S, Kondagunta GV, Bosl GJ, Sheinfeld J. Pathologic findings and clinical outcome of patients undergoing retroperitoneal lymph node dissection after multiple chemotherapy regimens for metastatic testicular germ cell tumors. Cancer 2007;109:528-535.

14. Marina NM, Cushing B, Giller R, Cohen L, Lauer SJ, Ablin A, Weetman R, Cullen J, Rogers P, Vinocur C, Stolar C, Rescorla F, Hawkins E, Heifetz S, Rao PV, Krailo M, Castleberry RP. Complete surgical excision is effective treatment for children with immature teratomas with or without malignant elements: A Pediatric Oncology Group/Children's Oncology Group Intergroup Study. J Clin Oncol 1999;17:2137-2143.

15. Gobel U, Calaminus G, Schnedier ST, Koch S, Teske C, Harms D. The malignant potential of teratomas in infancy and childhood: The MAKEI experience in non-testicular teratoma and implications for a new protocol. Klin Padiatr 2006;218:309-314.

16. Faure-Conter C, Rocourt N, Sudour-Bonnange H, Vérité C, Martelli H, Patte C, Frappaz D, Orbach D. Pediatric germ cell tumors. Bull Cancer 2013;100:381-391.

17. Göbel U, Calaminus G, Blohm M, Booss D, Felberbauer F, Hofmann U, Holschneider AM, Willnow U, Harms D. Extracranial non-testicualar teratoma in childhood and adolescence: Introduction of a risk score for stratification of therapy. Klin Padiatr 1997;4:209-234.

18. Göbel U, Calaminus G, Engert J, Kaatsch P, Gadner H, Bökkerink JP, Hass RJ, Waag K, Blohm ME, Dippert S, Teske C, Harms D. Teratomas in infancy and childhood. Med Pediatr Oncol 1998;1:8-15.

19. Rescorla FJ. Pediatric germ celltumors. Sem Pediatr Surg 2012;21:51-60.

20. Frazier LA, Weldon C, Armatruda J. Fetal and neonatal germ cell tumors. Semin Fetal Neonatal Med 2012;17:222-230

21. Cecchetto G, Riccipetitoni G, Inserra A, Esposito C, Michelazzi A, Ruggeri G, Spinelli C, Lima M. Italian Group of Pediatirc Surgical Oncology. Minimally-invasive surgery in paediatric oncology: Proposal of recommendations. Pediatr Med Chir 2010;32:197-201.

22. Gobbi D, Fascetti Leon, Aquino F, Melchionda A, Lima F. Metachronous bilateral ovarian teratoma: A germ-line familial disorder and review of surgical management options. J Pediatr Adolesc Gynecol 2013;26:105-107.

23. Taskinen S, Urtane A, Fagerholm R, Lohi J, Taskinen M. Metachronous benign ovarian tumors are not uncommon in children. J Pediatr Surg 2014;49:543-545.

24. Nezhat C, Kotikela S, Mann A, Hajhosseini B, Veeraswamy A, Lewis M. Familial cystic teratomas: Four case reports and review of the literature. J Minim Invasive Gynecol 2010;17:782-786.

25. Caspi B, Lerner-Geva L, Dahan M, Chetrit A, Modan B, Hagay Z, Appelman Z. A possible genetic factor in the pathogenesis of ovarian dermoid cysts. Ginecol Obstet Invest 2003:56:203-206.

26. De Backer A, Madern GC, Hakvoort-Cammel FG, Haentjend P, Oosterhuis JW, Hazebroek FW. Study of the factors associated with recurrence in children with sacrococcygeal teratoma. J Pediatr Sur 2006; $41: 173-181$

27. Logothetis CJ, Samuel ML, Trinidale A, Johnson DE. The growing teratoma syndrome. Cance 1982;50:1629-3529

28. Calaminus G, Schneider DT, Weissbach L, Schönberger S, Okpanyl V, Leuschner I, Poremba C, Göbel U. Survival after antiangiogenetic therapy and surgery in a wide spread growing teratoma originating from a testicular mixed malignant germ cell tumor. Klin Padiatr 2009;22:136-140. 